\title{
Integrable $\mathrm{KdV}$ systems: Recursion operators of degree four
}

\author{
Metin Gürses ${ }^{a}$, Atalay Karasu ${ }^{b}$ \\ a Department of Mathematics, Faculty of Sciences, Bilkent University, 06533 Ankara, Turkey \\ b Department of Physics, Faculty of Arts and Sciences, Middle East Technical University, 0653I Ankara, Turkey
}

Received 19 January 1998; revised manuscript received 19 November 1998; accepted for publication 27 November 1998

Communicated by A.P. Fordy

\begin{abstract}
The recursion operator and bi-Hamiltonian formulation of the Drinfeld-Sokolov system are given. (C) 1999 Elsevier Science B.V.
\end{abstract}

Recently [1,2] we have given a subclass of the coupled system of Korteweg-de Vries ( $\mathrm{KdV}$ ) equations. These systems of equations are mainly classified, if they are integrable, with respect to a pair of numbers $(m, n)$. Here the numbers $m$ and $n$ are respectively the highest powers of the operators $D$ and $D^{-1}$ in the recursion operator, $\mathcal{R}$. The type $(2,1)$ has been extensively studied by several authors [111]. The Svinolupov-Jordan KdV system [12,13] corresponds to the type $(2,2)$ and some mixed cases were considered quite recently [14]. In this work we start to consider the type $(4,1)$ for $N=2$. Our preliminary classification includes the systems: Hirota-Satsuma [15,4,16-21], Boussinesq [22-24], and Drinfeld-Sokolov (DS) systems [25,26]. The first two systems are well known to be integrable, that is they admit a recursion operator and also a bi-Hamiltonian structure. The latter one admits a Lax pair $[25,27]$ and its Painlevé truncated Backlund transformations are studied by Tian and Gao [28].

Here we show that the DS system admits a hereditary recursion operator and hence results out of a Hamiltonian pair. We consider a system of $N$ nonlinear equations which is called a coupled KdV system, $q_{t}^{i}=b_{j}^{i} q_{x x x}^{j}+s_{j k}^{i} q^{j} q_{x}^{k}+\chi_{j}^{i} q_{x}^{j}$,

where $i, j, k=1,2, \ldots, N, q^{i}$ are functions depending on the variables $x, t$, and $b_{j}^{i}, s_{j k}^{i}$ and $\chi_{j}^{i}$ are constants. Here we use the Einstein convention, i.e. repeated indices are summed up over $1-N$. The part containing the terms $\left(b_{j}^{i}, s_{j k}^{i}\right)$ will be called the principal part of the system of $\mathrm{KdV}$ equations (1). In the classification of the above system (1) singular and non-singular behavior of the matrix $b_{j}^{i}$ plays an essential role [2]. The system is called degenerate if $b$ is singular, i.e. $\operatorname{det}\left(b_{j}^{i}\right)=0$, otherwise it is called non-degenerate. The Hirota-Satsuma system is an example of a nondegenerate and the Boussinesq system is an example of a degenerate case.

We propose that the recursion operator (if it exists) of the system of equations in (1) takes the form

$$
\begin{aligned}
& \mathcal{R}_{j}^{i}=a_{j}^{i} D^{4}+D_{j k}^{i} q^{k} D^{2}+t_{j}^{i} D^{2}+\xi_{j}^{i} D+c_{j k}^{i} q_{x}^{k} D \\
& \quad+R_{j k}^{i} q_{x x}^{k}+S_{j l m}^{i} q^{l} q^{m}+Q_{j k}^{i} q^{k}+b_{j k}^{i} q_{x x x}^{k} D^{-1} \\
& \quad+M_{j l m}^{i} q^{l} q_{x}^{m} D^{-1}+N_{j l m}^{i} q_{x}^{l} D^{-1} q^{m}+P_{j k}^{i} q_{x}^{k} D^{-1} \\
& \quad+w_{j}^{i},
\end{aligned}
$$

where $D$ is the total $x$-derivative, $D^{-1}$ is the inverse operator and all parameters are constants. In this work 
we consider the system of equations (1) admitting recursion operators $(2)$ of the irreducible $(4,1)$-type. A recursion operator will be called irreducible if it is not possible to write it as $\mathcal{R}_{j}^{i}=\tilde{R}_{k}^{i} \tilde{R}_{j}^{k}$, where $\tilde{R}_{j}^{i}$ is the recursion operator of the type $(2,1)$ given by $\tilde{R}_{j}^{i}=b_{j}^{i} D^{2}+a_{j k}^{i} q^{k}+c_{j k}^{i} q_{x}^{k} D^{-1}$. KdV systems admitting reducible recursion operators of the type $(4,1)$ belong to the class studied recently [2].

Here we shall not give a systematic classification of this system for all $N$. For $N=2$ we present the recursion operator and bi-Hamiltonian formulation of the DS system. This system is a non-degenerate but contains a nontrivial linear term $q_{x}^{i}$. It is given in the following form,

$u_{t}=-u_{x x x}+6 u u_{x}+6 v_{x}, \quad v_{t}=2 v_{x x x}-6 u v_{x}$.

We find that the recursion operator $\mathcal{R}$ of this system is

$\mathcal{R}=\left(\begin{array}{ll}\mathcal{R}_{0}^{0} & \mathcal{R}_{1}^{0} \\ \mathcal{R}_{0}^{1} & \mathcal{R}_{1}^{1}\end{array}\right)$

with

$$
\begin{aligned}
& \mathcal{R}_{0}^{0}=D^{4}-8 u D^{2}-12 u_{x} D-8 u_{x x}+16 u^{2}+16 v \\
& \quad+\left(-2 u_{x x x}+12 u u_{x}+12 v_{x}\right) D^{-1}+4 u_{x} D^{-1} u \\
& \mathcal{R}_{1}^{0}=-10 D^{2}+8 u+4 u_{x} D^{-1} \\
& \mathcal{R}_{0}^{1}=10 v_{x} D+12 v_{x x}+\left(4 v_{x x x}-12 u v_{x}\right) D^{-1} \\
& \quad+4 v_{x} D^{-1} u \\
& \mathcal{R}_{1}^{1}=-4 D^{4}+16 u D^{2}+8 u_{x} D+16 v+4 v_{x} D^{-1} .
\end{aligned}
$$

Now it can be shown that this recursion operator satisfies the hereditary property [29]. Furthermore, it admits the factorization [30] $\mathcal{R}_{j}^{i}=\left(\theta_{2}\right)^{i k}\left(\theta_{1}^{-1}\right)_{k j}$, where

$\theta_{1}=\left(\begin{array}{cc}0 & \frac{1}{2} D \\ \frac{1}{2} D & \frac{1}{4} D^{3}-\frac{1}{2}(D u+u D)\end{array}\right)$

and

$\theta_{2}=\left(\begin{array}{cc}\theta_{2}^{00} & \theta_{2}^{01} \\ \theta_{2}^{10} & \theta_{2}^{11}\end{array}\right)$

with

$$
\begin{aligned}
& \theta_{2}^{00}=-5 D^{3}+4 u D+2 u_{x}, \\
& \theta_{2}^{01}=-2 D^{5}+4\left(D^{3} u+u D^{3}\right)+4 D u_{x} D+4 u_{x} D^{2} \\
& \quad+6 D v+2 v D,
\end{aligned}
$$

$$
\begin{aligned}
& \theta_{2}^{10}=-2 D^{5}+8 u D^{3}+4 u_{x} D^{2}+2 D v+6 v D, \\
& \theta_{2}^{11}=-D^{7}+4 u D^{5}+2 u_{x} D^{4}+2 D^{4}\left(u_{x}+2 u D\right) \\
& \quad+2 v D^{3}+2 D^{3} v-8 u D^{2}\left(u_{x}+2 u D\right) \\
& \quad-4 u_{x} D\left(u_{x}+2 u D\right)-8 v\left(u_{x}+2 u D\right)-8 u v_{x} .
\end{aligned}
$$

Following the procedure of Ref. [24] one can casily show that both differential operators are skew-adjoint and satisfy the Jacobi identities. Moreover they constitute a compatible pair. Hence the system (3) can be written in a bi-Hamiltonian form. Using the compatible Hamiltonian operators (5) and (6), we have

$\left(\begin{array}{l}u \\ v\end{array}\right)_{,}=\theta_{1}\left(\begin{array}{c}\frac{\delta \mathcal{H}_{1}}{\delta u} \\ \frac{\delta \mathcal{H}_{1}}{\delta v}\end{array}\right)=\theta_{2}\left(\begin{array}{c}\frac{\delta \mathcal{H}_{0}}{\delta u} \\ \frac{\delta \mathcal{H}_{0}}{\delta v}\end{array}\right)$,

associated with the Hamiltonian functionals

$$
\begin{aligned}
& \mathcal{H}_{0}[u, v]=\int\left(\frac{1}{2} u^{2}+v\right) \mathrm{d} x, \\
& \mathcal{H}_{1}[u, v]=\int\left[\frac{1}{2} u_{x x}^{2}-\frac{5}{2} u^{2} u_{x x}+\frac{5}{2} u^{4}+2 u_{x} v_{x}\right. \\
& \left.\quad+6\left(u^{2} v+v^{2}\right)\right] \mathrm{d} x .
\end{aligned}
$$

There thus exists a whole hierarchy of conservation laws and commuting symmetries (flows) for the DS system.

We have a second commuting hierarchy originated from the translational symmetry which may be formulated in a bi-Hamiltonian structure

$$
\left(\begin{array}{l}
u \\
v
\end{array}\right)_{t}=\left(\begin{array}{l}
u \\
v
\end{array}\right)_{x}=\theta_{1}\left(\begin{array}{c}
\frac{\delta \hat{\mathcal{H}}_{1}}{\delta u} \\
\frac{\delta \hat{\mathcal{H}}_{1}}{\delta v}
\end{array}\right)=\theta_{2}\left(\begin{array}{c}
\frac{\delta \hat{\mathcal{H}}_{0}}{\delta u} \\
\frac{\delta \hat{\mathcal{H}}_{0}}{\delta v}
\end{array}\right),
$$

where

$\hat{\mathcal{H}}_{0}[u, v]=\int \frac{1}{2} u \mathrm{~d} x$,

$\hat{\mathcal{H}}_{1}[u, v]=\int\left(\frac{1}{2} u_{x}^{2}+2 u v+u^{3}\right) \mathrm{d} x$,

are both conserved.

The interesting point here is that the linear term $v_{x}$ in this system is nontrivial. In the case of the $\mathrm{KdV}$ systems admitting recursion operators of type $(2,1)$, the linear terms $\chi_{j}^{i} q_{x}^{j}$ are not essential in the study 
of the integrability of these systems. This is based on the theorem given in Ref. [2]. It states that a $\mathrm{KdV}$ system with linear first derivative terms is integrable if and only if its principal part (system without the linear first derivative terms) is integrable. In the case of the $\mathrm{KdV}$ systems admitting recursion operators of type $(4,1)$ this theorem is not valid anymore.

The authors would like to thank an anonymous referee for valuable remarks. We also thank Professor V. Sokolov for his constructive comments. This work is partially supported by the Scientific and Technical Research Council of Turkey (TUBITAK) and by the Turkish Academy of Sciences (TUBA).

\section{References}

[1] M. Gürses, A. Karasu, Phys. Lett. A 214 (1996) 21.

[2] M. Gürses, A. Karasu, J. Math. Phys. 39 (1998) 2103.

13] M. Ito, Phys. Lett. A 91 (1982) 335.

14] B. Fuchssteiner, Prog. Theor. Phys. 68 (1982) 1082.

[5] W.X. Ma, B. Fuchssteiner, Phys. Lett. A 213 (1996) 49

$[6]$ B.A. Kupershmidt, J. Phys. A 18 (1985) L571.

[7] M. Antonowicz, A.P. Fordy, Physica D 28 (1987) 345.

[8] C. Athorne, A.P. Fordy, J. Phys. A 20 (1987) 1377.
[9] Q.P. Liu, J. Math. Phys. 35 (1994) 816.

[10] P.J. Olver, P. Rosenau, Phys. Rev. E 53 (1996) 1900.

[11] A.S. Fokas, Q.M. Liu, Phys. Rev. Lett. 77 (1996) 2347.

[12] S.I. Svinolupov, Theor. Mat. Fiz. 87 (1991) 391.

[13] S.I. Svinolupov. Functional Anal. Appl. 27 (1993) 257.

[14] P.J. Olver, V.V. Sokolov, Commun. Math. Phys. 193 (1998) 245.

[15] R. Hirota, J. Satsuma, Phys. Lett. A 85 (1981) 407.

[16] R. Dodd, A.P. Fordy, Phys. Lett. A 89 (1982) 168.

[17] W. Oevel, Phys. Lett. A 94 (1983) 404.

[18] R.N. Aiyer, Phys. Lett. A 93 (1983) 368.

[19] G. Wilson, Phys. Lett. A 89 (1982) 332.

[20] D. Levi, Phys. Lett. A 95 (1983) 7.

[21] X. Geng, Y. Wu. J. Math. Phys. 38 (1997) 3069.

[22] H.P. McKean, Boussinesq's Equation as a Hamiltonian System, Topics in Functional Analysis, Adv. in Math. Suppl. Stud., Vol. 3 (Academic Press, New York, 1978) pp. 217226.

[23] M. Adler, Inven. Math. 50 (1979) 219.

[24] P.J. Olver, Applications of Lie Groups to Differential Equations, 2nd Ed., Graduate Texts in Mathematics, Vol. 107 (Springer, New York, 1993).

[25] V.G. Drinfeld, V.V. Sokolov, Proc. S.L. Sobolev Seminar, Novosibirsk, Vol. 2 (1981) pp. 5-9 (in Russian)

[26] V.G. Drinfeld, V.V. Sokolov, J. Sov. Math. 30 (1985) 1975.

[27] O.I. Bogoyavlenskii, Russian Math. Surveys 45 (1990) 1.

[28] B. Tian, Y. Gao, Phys. Lett. A 208 (1995) 193.

[29] B. Fuchssteiner, Prog. Theor. Phys. 65 (1981) 861.

[30] B. Fuchssteiner, A.S. Fokas, Physica D 4 (1981) 47 . 